\title{
Improving Adherence in Automated e-Coaching
}

\section{A Case from Insomnia Therapy}

\author{
Robbert Jan Beun ${ }^{1}$, Willem-Paul Brinkman ${ }^{2}$, Siska Fitrianie ${ }^{1}$, Fiemke Griffioen- \\ Both $^{1}$, Corine Horsch ${ }^{2}$, Jaap Lancee ${ }^{3}$, Sandor Spruit ${ }^{1}$ \\ ${ }^{1}$ Utrecht University, Utrecht, The Netherlands \\ \{r.j.beun, s.fitrianie, f.griffioen-both, a.g.l.spruit\}@uu.nl \\ ${ }^{2}$ Delft University of Technology, Delft, The Netherlands \\ w.p.brinkman@tudelft.nl, corinehorsch@gmail.com \\ ${ }^{3}$ University of Amsterdam, Amsterdam, The Netherlands \\ j.lancee@uva.nl
}

\begin{abstract}
Non-adherence is considered a problem that seriously undermines the outcome of behavior change therapies, in particular of self-help therapies delivered without human interference. This paper presents the design rationale behind a computer system in the domain of adherence enhancing strategies in automated e-coaching. A variety of persuasive strategies is introduced and implemented in a mobile e-coaching system in the domain of insomnia therapy. The system integrates two types of interface elements, i.e. dedicated tools and natural language conversation, to simplify therapy related activities and to include social strategies to improve motivation. We focus on the crucial role of communication and adaptation.
\end{abstract}

Keywords. e-coaching $\bullet$ adherence $\bullet$ persuasive strategies $\bullet$ insomnia therapy $\bullet$ conversation $\bullet$ tools $\bullet$ behavior change systems

\section{Introduction}

One of the first software programs that automatically mimicked the behavior of a therapist is the digital Rogerian psychoanalyst 'Eliza' [26]. Eliza simulates a simple question-answer conversation in natural language by rephrasing many of the patient's statements into questions. The program showed that a digital therapist could be implemented in a computer system that has strong positive emotional effects with relatively simple means.

Since the appearance of Eliza the world changed radically. In the digital world, powerful mobile systems support the majority of the population with a wide range of activities; in health care, awareness increased that our health can be positively influenced by our behavior and activities. In the field of consumer health care, these developments resulted in a variety of products, ranging from various types of monitor-

In: Persuasive Technology. Proceedings of the $11^{\text {th }}$ Int. Conf., PERSUASIVE 2016, Salzburg, Austria, April 5-7, 2016, LNCS, Volume 9638. Springer International Publishing. 276-287. DOI: 10.1007/978-3-319-31510-2_24 
ing systems to self-help apps that aim to change an individual's behavior and cognition. Today over 100,000 health apps exist in the Apple and Google stores that support all types of health related activities, but a major problem is that a proliferation of consumer health care applications arises regardless of real user needs [21]. Potentially, we now have the knowledge and the technology to build interventions and coaching principles in smartphones that are far beyond the capabilities of Weizenbaum's Eliza, but the design of these systems should be preceded by careful analysis of the coaching process.

In this paper, we present the design rationale behind a fully automated coaching system that supports an intervention for behavior change in the domain of insomnia therapy - we refer to this system as the 'SleepCare-system'. The system behaves as a digital agent, the so-called e-coach, and integrates various types of interaction, in particular natural language conversation and graphical interaction with various tools. We focus in particular on the requirements and the generic elements in the design that were included to improve exercise adherence.

We first elaborate on the coaching process and some existing e-coaching implementations. We then turn to the domain of the application, insomnia therapy, and the problem of exercise adherence within the domain. Next, we discuss various adherence enhancing strategies to improve behavior change. We then turn to the design elements of the system that support these strategies and consider some aspects of testing. We finish this paper with a discussion about the relevant design elements.

\section{Coaching and e-Coaching}

Central to most definitions of coaching is that coaching is a goal-oriented activity to help people enhance aspects of both their personal and professional lives by fostering self-directed learning through collaborative goal setting, action planning and feedback [14]. Assumptions in the coaching process are the absence of serious mental health problems [6] and the notion that the client - or coachee - is resourceful and motivated to engage in finding solutions [1] [17]. In the coaching process, two learning dimensions are essential: (1) learning through individual subjective experiences and (2) learning as a social and collaborative practice. The first dimension refers to the experiential and action oriented process of the coachee; the second refers to the idea of a collaborative dialogue that unfolds between the coaching parties and where the coachee learns in interaction with the coach.

Since current society and daily lives of people are highly contextual and characterized by a growing degree of uncertainty, a coach will be cautious in offering solutions. What counts as a solution for one person, not necessarily counts as a solution for another. Therefore, coaching involves a collaborative approach and respect of the coachee's autonomy. Personalization, contextualization and frequent adaptation are necessary prerequisites of the coaching process. In that respect, the role of a coach seems an excellent candidate for a digital agent that supports automated self-help therapies; we will refer to these systems as e-coaches. 
Examples of e-coaches that apply methods from behavior medicine cover a wide range of therapy domains, ranging from obesity to depression and insomnia treatment. In [5], for example, an e-coach was developed to support overweight people improving their lifestyle. The e-coach is able to help motivated participants adhere to the program and lose weight. In [7], an e-coach is described that offers a fully automated treatment for depression, based on behavioral activation, a form of psychotherapy. In [9] an e-coach is described that offers an insomnia treatment in six weekly sessions. The authors successfully tested the intervention against a placebo and treatment as usual in a randomized controlled trial.

Previous studies mainly focus on one specific domain. An important exception to domain specificity is the work described in [4] who describe a theory-driven computational model to deliver health behavior change counseling. The authors present an ontology containing concepts from behavioral medicine and communication. Various models are introduced that enable the representation of knowledge about, for instance, the user and the underlying theory of the intervention. We also aim at developing a reusable mobile framework based on generic coaching principles, but in contrast to [4] who focuses on modeling counseling knowledge from which dialogue actions can be inferred, our perspective starts from communication and cybernetics theory, and is based on concepts such as alignment, negotiation, evaluation, feedback and adaptation. This enables us to include persuasive strategies such as tunneling, adaptation, reduction and increase of motivation. The insomnia domain will be used as a proof of concept.

\section{Insomnia and Insomnia Therapy}

Insomnia is a sleep disorder with a high prevalence (about $10 \%$ of the population) and can have severe individual and societal consequences (e.g., concentration problems, increased risks of accidents, depression); people with insomnia have difficulty initiating and/or maintaining sleep. Today, it is widely accepted that behavior change treatments such as Cognitive Behavior Therapy (CBT) produce sustainable positive changes in the condition of insomnia [22].

CBT for insomnia (CBT-I) offers a variety of exercise types that differ in aim and properties [23]: sleep restriction, stimulus control, relaxation, cognitive therapy and sleep hygiene. Sleep restriction involves curtailing the time spent in bed to stabilize the sleep pattern and lengthening sleep time as sleep efficiency improves. Stimulus control aims at restoring the coachee's positive association of the bed and the bedroom with sleep. Relaxation training involves methods aimed at reducing somatic tension. Cognitive therapy aims at changing dysfunctional beliefs and attitudes. Sleep hygiene education aims to make the person aware of practices and environmental factors that may either be detrimental or beneficial for sleep. Treatment protocols usually take between 6 and 10 weekly consultation sessions. The actual intervention is preceded by a one- or two-week baseline sleep diary monitoring period. 


\section{$4 \quad$ Exercise Adherence}

Central in self-management approaches to behavior change for health - and CBT-I is no exception to this - is the idea that the individual invests a reasonable amount of effort and time in the activities. However, people have many reasons not to perform the assignments: lack of energy, motivation and willpower, mistrust, procrastination, simply forgetting, etcetera. As a result, low adherence rates are an ever present and complex problem, and seriously undermine the outcome of CBT [25]. In [15], it has been shown that the average treatment adherence for technology mediated insomnia treatments is approximately $52 \%$, meaning that almost half of the people did not finish the treatment.

It may even be expected that in reality these rates are considerably lower. First, since these results were based on self-reports and user logs, the reports by the participants may be overrated and based on a positive answer bias. Second, adherence is a complex notion and has no clear-cut boundaries (e.g., total time in bed, number of relaxation exercises per day, completion of the assignments). Low adherence rates can be expected in particular with exercise types that require strong willpower.

Preventing the process of a downward motivation spiral and adequately responding to it is a mandatory element in any behavior change intervention. For that, we should realize that the exercises in insomnia treatment include a large variation of activities in terms of actual content, duration, timing, frequency, presentation and intensity (cf. [12]). Some of these activities take time and should be performed on a daily or weekly basis such as relaxation exercises or consults. Sleep restriction and stimulus control require a great deal of willpower and can even be dangerous, but may have high treatment effects if well adhered to. The sleep hygiene exercise may require changing habits such as refrain from drinking coffee or alcohol before bedtime; in other cases, the exercise requires a one-time behavior such as cleaning the bedroom or changing the bedroom temperature. Improving adherence to this variation of therapy elements requires a well-dosed combination of various adherence enhancing strategies.

\section{$5 \quad$ Adherence Enhancing Strategies}

\subsection{Tunneling, Feedback and Adaptation}

Basically, every CBT-coach offers evidence based behavior change techniques that should be experienced by the coachee for a particular period of time. The behavior in the CBT-I domain consists of introducing or changing activities in the coachee's daily life such as filling in a sleep diary, having a conversation for consultation or changing bedtime; in other cases, activities and habits are discouraged such as drinking coffee or alcohol just before bedtime. In other words, a coach tunnels therapy related activities: it determines the exercise types, the properties of these exercises and communicates this information to the coachee. We call this process tunneling (cf. [11]).

In contrast to self-help books, a human coach adds an important quality to the tunneling process: a feedback loop. To cope with the many uncertainties, a frequent 
feedback loop is included to reshape the offered techniques and the communication about these techniques. Hence, the information flow between coach and coachee not only pertains to tunneling (e.g., 'Go to bed at 12', 'Fill in your sleep diary before 8'), but also to the adaptation process. A human coach observes, asks questions, negotiates, agrees, aligns and tailors the exercises to the multifarious coachee characteristics such as age, habits, preferences, intermediary results and experiences [3]. Adaptation is a crucial element in the tunneling process. A person who, for whatever reason, is unable to go to bed at twelve and repeatedly hears from her coach that she should go to bed at twelve, most likely decides that the therapy is not applicable and may even consider the coach as ignorant or unreliable. This is one of the reasons that the results of many self-help books are rather disappointing [24]: they are targeted at large user groups and do not offer information or exercises tailored to the individual.

\subsection{Persuasive Strategies}

Coaches also apply a range of behaviors to support the behavior change: they motivate, encourage, challenge, and explain; they show progress and confront with discrepancies in real and committed behaviors. In that sense, coaching is a constant process of tunneling, tailoring and support. We refer to a collection of coherent activities that support the process of behavior change as a persuasive strategy. In line with [13] and [20], we assume that persuasive strategies aim at increasing the ability and/or motivation of the coachee with respect to the intended behavior change. Loosely speaking, improving ability implies that the coachee may decrease the amount of effort that should be put into the therapy; improving motivation implies that the coachee is willing to put more effort into the therapy. The distinction between the two concepts is not always that clear, however.

We assume that the coachee is located in a complex multidimensional space, the current state, and aims to achieve another complex space, the desired state. In insomnia therapy, both states can be expressed in concrete values for sleep variables such as total sleeping time and quality of sleep. In the desired state, the coachee has improved' on at least some of these values. To stress the importance of the difference between the use of tools and language (cf. [11]), persuasive strategies are now classified in two categories (cf. [16]): (1) polishing strategies and (2) meta-level strategies.

ad 1. The road to the desired world is full of obstacles. An important function of the coach is to take away these obstacles and polish the way to success. Metaphorically speaking, the coach offers a slide to improve the flow of activities that have to be performed to achieve the desired world. It may simplify behavior and help to avoid boredom and stress; it may include beauty or a positive user experience to attract attention and willingness to participate. In practice, this may imply that the coach offers tools, e.g., an electronic sleep diary, an agenda, relaxing music or a game. This is also related to what is often called 'reduction' and 'nudging'. In insomnia therapy, for instance, a tool may be implemented that automatically lowers the intensity or changes the color of the light to induce homeostatic processes in the body for bedtime preparation. 
ad 2. The meta-level adds a symbolic level to the previous category. It is what we often call 'social influence' and may, for instance, be represented in the interface as an embodied character that uses natural language dialogue and nonverbal signs. The coach is now able, for instance, to communicate that 'if the coachee performs an activity, then the coachee will be rewarded'. The use of symbols enables the coach to approve or disapprove the coachee's behavior, to communicate about the road to the desired state and to explain matters in terms of cause and consequence. The metalevel also enables to negotiate the intensity of the intervention, or to show some type of authority (e.g., 'This is an evidence based intervention'). Including the meta-level enables us to incorporate social elements that have a high impact on a person's behavior: collaboration, transparency, competition, trust, commitment, reciprocity (see e.g., [8]) and accompanying speech acts such as promise, praise, welcome, bid, finish the conversation, etcetera. The meta-level substantially increases the expressivity of the coaching system; in practice, the levels are constantly intertwined.

In CBT-I two types of activities can be distinguished: a. main activities that have to be experienced by the coachee such as sleep restriction and b. supporting activities such as activity scheduling. To restructure the coachee's sleep architecture, it is important to experience sleep pressure, but it is unimportant to experience calculating sleep efficiency. Consequently, all activities that are part of CBT-I and that do not have to be experienced by the coachee can be simplified. In CBT-I, for instance, sleep data can be registered by means of an electronic diary instead of paper and pencil notation; calculations of sleep efficiency can be automated and integrated in a proposal for wake up and bedtimes. In the ideal case, supporting activities are fully automated. Because main activities, such as decreasing total bedtime, should be experienced, it is important to increase motivation for these types of activities.

To conclude, an e-coach that is intended to offer an intervention for behavior change should include a variety of adherence enhancing strategies that aim at:

- tunneling the intended activities,

- adaptation of exercises and interaction,

- reduction by simplification of supporting activities,

- increasing motivation to perform main activities (cf. [19]).

We now turn to the design of the SleepCare-system and explain how these requirements were applied in the system.

\section{The SleepCare System}

\subsection{The Functionality of the e-Coach}

The SleepCare-system offers a therapy on an Android smartphone that includes a variety of CBT-I exercises: relaxation, sleep restriction and sleep hygiene. Sleep restriction has been chosen, because it is an effective element of CBT-I; relaxation and sleep hygiene were chosen because these exercises were relatively easily to implement and vary substantially from sleep restriction. Exercises consist of assignments 
that have to be carried out during the therapy. There are five types of assignments: going to bed, getting out of bed, filling in the sleep diary, doing a relaxation exercise and taking part in a consult. A consult is a conversational activity about various therapy related topics such as the introduction and evaluation of a particular exercise type.

In the therapy, three interaction stages are distinguished: opening, intervention and closure [3]. The goal of the opening phase is twofold: improving transparency through a process of alignment and establishing commitment to the therapy. In the intervention phase the actual therapy is conducted and the coachee is supposed to carry out the assignments. The closure phase starts when all assignments have been performed or when the coachee indicates the desire to withdraw. In this phase, the e-coach and coachee evaluate the offered therapy and may say goodbye.

\subsection{Tunneling the coachee's activities}

A fundamental element in the process of tunneling is the activity schedule that is introduced by the e-coach in one of the early introduction sessions. The primary task of the activity schedule is to keep track of the various assignments that have been or should be carried out during the therapy. All scheduled assignments result from an agreed contract between coach and coachee. The end time of the activities is usually unknown, but in practice the time interval of assignments is relatively short (e.g., between 1 and 16 minutes). Scheduled assignments may trigger the generation of reminders and other communication acts. Activities by the e-coach may also be triggered by non-scheduled events, in particular when the system detects violations of pre-defined 'constraints' such as obvious non-adherence of the coachee [3].
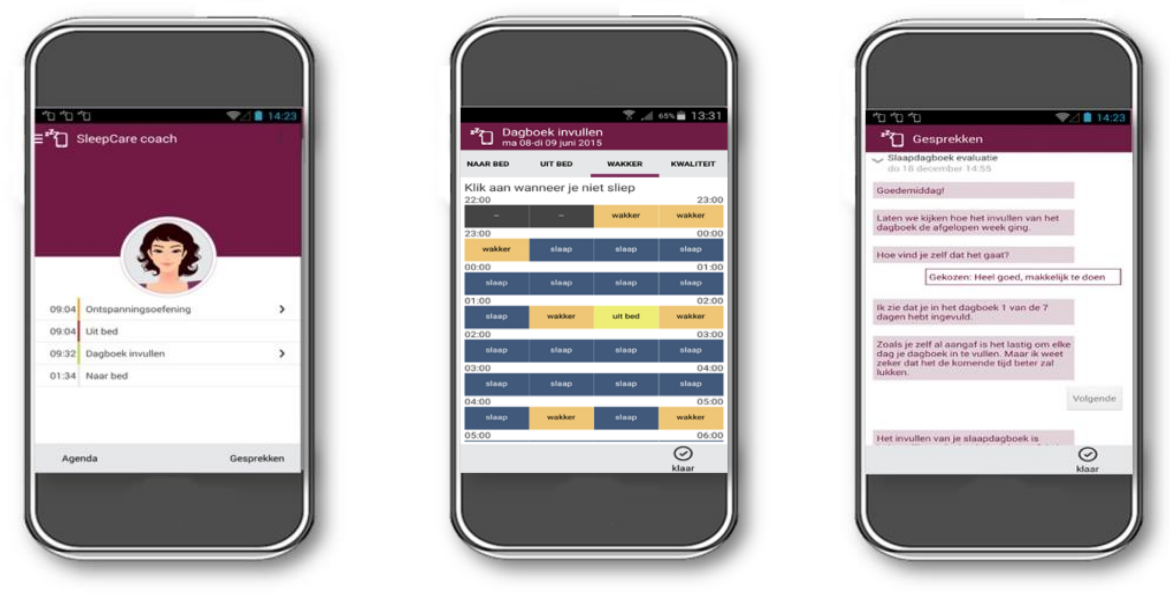

Fig. 1. The left picture depicts the home screen of the SleepCare-system and a part of the schedule tool; colored vertical lines indicate the performance status of the exercises. The middle and right picture show examples of the sleep diary tool and an evaluation dialogue about the use of the sleep diary. On top of the interface notification icons appear in the notification area (the small black beam). 
To observe the properties of the scheduled assignments, such as starting-time and performance status, there exists a corresponding interaction tool. The most important function of the tool is to display the coachee's previous commitments and adherence to the assignments. For that, the interaction tool not only displays the scheduled starting time and date in the past, present or future, but also the performance status of an assignment by a colored line in front: red means missed, orange means near missed, green means completed and grey means planned or actual. The left picture in Figure 1 shows a part of the interaction tool presented in the system's home screen. As a consequence, the tool not only enables a coachee to have a prompt overview of the future commitments, but also of the (non-)adhered assignments in the past. In that way, the schedule-tool not only tunnels the coachee's activities, but also refers to meta-level properties such as agreed commitments

\subsection{Feedback and Adaptation}

Starting from the opening phase, there is a constant activity of feedback and adaptation. From the e-coach's point of view, the goal of the opening phase is to construct a user model to adapt the therapy to the characteristics of the coachee. The adaptation process pertains to information at both the therapy and the communication level (e.g., sleep characteristics, age and name). Based on a set of exclusion criteria, the coach may also advise the coachee to withdraw from the therapy; the coachee, however, makes the final decision about continuation.

During the intervention phase, exercises always follow the same four steps [3]: 1. introduction, 2. planning and commitment, 3. task execution and 4. evaluation. In the introduction step, the exercise type is explained. In the planning and commitment step, e-coach and coachee aim at agreement about the exercise properties. During task execution the coachee performs the assignments. In the evaluation step, results are discussed and the exercise properties may, in deliberation with the e-coach, be adapted to the coachee's desires. In case of exercise adaptation, the process is repeated, starting from the planning and commitment phase. If no constraints are violated, the planning-evaluation cycle takes about one week. All exercise steps are included in the activity schedule, but in the course of the therapy, assignments may be added, rescheduled or even be removed in deliberation with the e-coach.

\subsection{The User Interface}

In the system, four categories of interface elements are distinguished: conversations, tools, notifications and the menu. Conversations enable the e-coach to perform metalevel natural language interaction activities such as introductions and weekly consults. Tools are relatively independent modules that are less language oriented and contain graphical elements such as buttons, wheels and tables; we included the schedule-tool, an electronic sleep diary and a relaxation tool. Notifications function as attention grabbers to start a particular activity and are displayed outside the system's normal user interface (see Figure 1 middle and right pictures). The menu offers access to the 
various tools, performed conversations, sleep exercises, advice and information, and general issues such as notification settings, privacy and safety.

Although simplistic compared to human F2F-conversations, the use of natural language interaction enables us to include a plethora of conversational structures that resemble well-known collaboration and persuasion strategies at the meta-level. As such, conversations are the central interaction concept in the system. They not only explain the assignments (what, how and why) and what can be expected (e.g., difficulties, results), but also function as an instrument for adaptation, shared commitment and explicit motivational support such as praise and encouragement.

To elucidate a conversational interaction, we briefly focus on one of the meta-level strategies used in the SleepCare-system: the establishment of a shared commitment by negotiation. An adapted version of a conversation about the sleep restriction exercise is shown in the dialogue fragment below (translated from Dutch; E: e-coach, C: coachee). In this fragment, the coachee options are given between brackets (selected options in bold); values of variables based on the e-coach's knowledge base are represented in italics. In this case, we have chosen a persuasive strategy of shared decision making, because sleep restriction is an intensive exercise and can even be dangerous in certain circumstances (e.g., [18]). The negotiation space depends on the user mod$\mathrm{el}$, in particular total bedtime and sleep efficiency, and a number of rules, for instance, total bedtime should never be shorter than 5 hours.

E Good morning John. This week we will start the sleep restriction exercise. You have to keep regular bed times and should shorten the total time in bed. Because you have less time to sleep, you will be more tired and that helps for a better sleep. Would you like to know more before we start?

C [Let's start] [What do I have to do?] [What can I expect?]

E We will now determine your bedtimes. On the basis of your sleep diary I suggest that the total bedtime will be 5 hours. Do you agree?

C [Yes] [I prefer longer][I don't want to do bedtime restriction]

E How long do you want to stay in bed?

C [5.30][6.00][6.30]

E You suggested 6.30 hours. The exercise works better when you stay in bed as long as your mean sleeping time last week. I therefore suggest that the total time in bed will be 5.30. Do you agree?

C [Yes][I prefer longer]

E $\quad \cdots$

To cover the main interaction needs in CBT-I, 18 different conversational types were included (e.g., intro, planning and commitment, evaluation). These conversations can be considered as tree like structures where selections of the coachee or the value of particular sleep and behavior data causes a branch. Half of them are scheduled based, and the others are constraint-based conversations. Conversations are generated using XML-based predefined templates for describing the discourse moves of the e-coach [10]. All conversations (including the branches) were evaluated by an expert in Dutch communication and a sleep therapist. 


\section{$6.5 \quad$ Testing}

The complex nature of the system and the application domain, the multimodal nature of the interaction, the large amounts of collected user knowledge and the many uncertainties in the use of mobile applications make the testing procedure a challenge. In particular, the variety in the circumstances of use is one of the main difficulties in the development of a smartphone application. Different versions of Android and smartphone manufacturers and changes thereof during use, network availability, unexpected user behavior and changes in the environment may cause a great deal of uncertainty in the outcome of the offered therapy. This not only requires that the system functions flawlessly, but also that the e-coach offers a therapy that is safe and in line with the advice of human therapists even under extreme circumstances.

For testing purposes, scenarios were written together with a sleep psychologist, making sure that the scenarios and user data covered the most common sleeping patterns and extremes on both ends as well. The scenarios were simulated and recorded in videos. A group of four sleep experts watched and commented on the behavior of the e-coach in the videos, providing us with information on the validity of the offered therapy. They focused in particular on the critical scenarios in which people sleep less than five hours per night.

Next, a pilot experiment was organized to test the SleepCare-system with intended users. 24 people asynchronously started using the app in their daily life and focused in particular on usability issues, the interaction with the e-coach and the circumstances of a possible system break down. Main success criteria for continuation were: a. no decrease in sleep efficiency and sleep quality, and b. no technical failure. The results gave us enough confidence in the system and the training program to execute a randomized controlled trial (RCT) as a next step in the research process.

\section{Discussion}

In this paper, strategies were discussed for enhancing adherence in behavior changing therapies delivered by digital systems. As a proof of concept, some of the strategies were implemented in an automated e-coaching system that supports insomnia therapy - the so-called SleepCare-system. An interface was realized that distinguishes between graphical tools and natural language conversations. Conversations enable us to include meta-level persuasive strategies based on social influence, such as commitment and shared decision making. We have stressed the importance of a design rationale and a careful analysis of the coaching process.

We briefly recapitulate the strategies discussed in this paper and their implementation in the SleepCare-system:

Tunneling: the e-coach should communicate the intended activities at the right moment in the right form. In SleepCare a sophisticated activity schedule, notifications and reminders were included.

Adaptation: exercises and interaction should be personalized and adapted to the circumstances. In SleepCare an explicit alignment phase and frequent feedback loops were included. 
Reduction: therapy related activities that do not have to be experienced can be supported by tools or automated. In SleepCare tools were included for handling sleep data (e.g., registration, overviews), scheduling and relaxation.

Increasing motivation: therapy related activities that must be experienced can be supported by increasing the coachee's motivation. Examples in SleepCare are: showing (non-)adherence, performance and progress, shared decision making, explicit user commitment, explanation of therapy and exercise rationale, and expectation management.

Our first activity for the future is experimental verification of the SleepCaresystem by means of the RCT. Next we will extend the adherence enhancing strategies to other domains and user groups, for example shift workers, and include automated sleep registration by non-obtrusive sensor information.

Finally, we may ask what we have learned since the appearance of Weizenbaum's digital therapist Eliza. We believe that in the future, consumers in e-health will not only be confronted with a variety of tools for monitoring health related characteristics and telecommunication with human caretakers, but also with automated e-coaches or e-doctors that direct a user's behavior. Computer systems aiming at behavior change will be equipped with adherence enhancing techniques to 'seduce' their users to do the therapy activities. This not only raises interesting philosophical questions, but also questions with respect to ethical design. Clearly, extensive testing is a mandatory element, but we also believe that explicit strategies should be included that protect the user's autonomy. We have the tools and the language, we now need the knowledge.

Acknowledgements. This research is supported by Philips and Technology Foundation STW, Nationaal Initiatief Hersenen en Cognitie NIHC under the Partnership programme Healthy Lifestyle Solutions.

\section{$8 \quad$ References}

1. Berg, I.K., Szabo, P.: Brief coaching for lasting solutions. NY: W.W. Norton (2005)

2. Beun, R.J.: Persuasive strategies in mobile insomnia therapy: alignment, adaptation, and motivational support. Personal and Ubiquitous Computing 17(6), 1187-1195 (2013)

3. Beun, R.J., Griffioen-Both, F., Ahn, R., Fitrianie, S. and Lancee, J. (2014). Modeling Interaction in Automated e-Coaching: A Case from Insomnia Therapy. In: $6^{\text {th }}$ Int. Conf. on Adv. Cognitive Technologies and Applications (2014)

4. Bickmore, T.W., Schulman, D., Sidner, C.L.: A reusable framework for health counseling dialogue systems based on behavioral medicine ontology. J. of Biomedical Informatics 44, 183-197 (2011)

5. Blanson Henkemans, O.A., van der Boog, P.J.M., Lindenberg, J., van der Mast, C.A.P.G. Neerincx, M.A., Zwetsloot-Schonk, B.J.H.M.: An online lifestyle diary with a persuasive computer assistant providing feedback on self-management. Technology \& Health Care 17(3), 253-257 (2009)

6. Bluckert, P.: The similarities and differences between coaching and therapy. Industrial \& Commercial Training 37(2), 91-96 (2005) 
7. Both, F., Cuijpers, P., Hoogendoorn, M., Klein, M.: Towards Fully Automated Psychotherapy for Adults: BAS - Behavioral Activation Scheduling via web and mobile phone. In: 3d Int. Conf. on Health Informatics 2010, 375-380 (2010)

8. Cialdini, R.B.: Influence: Science and Practice. New York: Pearson (1993)

9. Espie, C.A., Kyle, S.D., Williams, C., Ong, J.J., Douglas, N.J., Hames, P., Brown, J.S.L.: A Randomized, Placebo-Controlled Trial of Online Cognitive Behavioral Therapy for Chronic Insomnia Disorder Delivered via an Automated Media-Rich Web Application. Sleep 35(6), 769-781 (2012)

10. Fitrianie, S., Griffioen-Both, F., Spruit, S., Lancee, J., Beun, R.J.: Automated Dialogue Generation for Behavior Intervention on Mobile Devices. Procedia Computer Science 63, 236-243 (2015)

11. Fogg, B.J.: Persuasive Technology: Using computers to change what we think and do. San Francisco: Morgan Kaufmann (2002)

12. Fogg, B.J.: The Behavior Grid: 35 ways behavior can change. In: Persuasive '09, Claremont, California (2009a)

13. Fogg, B.J.: A behavioral model for persuasive design. In Persuasive '09, Claremont, California (2009b)

14. Greene, J., Grant, A.M.: Solution-focused coaching: Managing people in a complex world. London: Momentum Press (2003)

15. Horsch, C.H.G., Lancee, J., Beun, R.J., Neerincx, M.A., Brinkman, W.P.: Adherence to technology-mediated insomnia treatment: A meta-analysis, interviews with users, and focus groups with users and experts. J. of Med. Internet Research 17(9) (2015)

16. Horsch, C.H.G., Brinkman, W.P., van Eijk, R.M., Neerincx, M.A.: Towards the usage of persuasive strategies in a virtual sleep coach. In: UKHCI 2012 Workshop on People, Computers and Psychotherapy (2012)

17. Hudson, F.M.: The handbook of coaching. San Francisco: Jossey-Bass (1999)

18. Kyle, S.D., Miller, C.B., Rogers, Z., Sirwardena, A.: Sleep restriction therapy for insomnia is associated with reduced objective total sleep time, increased daytime somnolence, and objectively-impaired vigilance: implications for the clinical management of insomnia disorder. Sleep 37(2), 229-237 (2014)

19. Lancee, J., van den Bout, J., Sorbi, M.J., van Straten, A. Motivational support provided via email improves the effectiveness of internet-delivered self-help treatment for insomnia: A randomized trial. Behaviour Research and Therapy 51, 797-805 (2013)

20. Michie, S., van Stralen, M.M., West, R.: The behavior change wheel: A new method for characterizing and designing behavior change interventions. Implementation Science 6(42) (2011)

21. Middelweerd, A., Mollee, J.S., van der Wal, N., Brug, J., te Velde, S.J.: Apps to promote physical activity among adults: a review and content analysis. Int. J. of Behavioral Nutrition and Physical Activity 11(97) (2014)

22. Morin, C.M., Bootzin, R.R., Buysse, D.J., Edinger, J.D., Espie, C.A., Lichstein, K.L.: Psychological and behavioral treatment of insomnia: Update of the recent evidence (19982004), Sleep 29, 1398-1414 (2006)

23. Morin, C.M., Espie, C.A.: Insomnia. A clinical guide to assessment and treatment. New York: Springer (2003)

24. Van Straten, A., Cuijpers, P., Smit, F., Spermon, M., Verbeek, I.: Self-help treatment for insomnia through television and book: a randomized trial. Patient Educ. Couns. 74(1), 2934 (2009) 
25. Vermeire, E., Hearnshaw, H., Van Royen, P., Denekens, J.: Patient adherence to treatment: three decades of research. A comprehensive review. J. of Clinical Pharmacy and Therapeutics 26, 331-342 (2001)

26. Weizenbaum, J.: ELIZA - A computer program for the study of natural language communication between man and machine. Communication of the ACM 9(1), 36-45 (1966) 